\title{
Improving Naive Bayes for Regression with Optimised Artificial Surrogate Data
}

\author{
Michael Mayo and Eibe Frank \\ Department of Computer Science \\ University of Waikato \\ Hamilton, New Zealand
}

November 29, 2018

\begin{abstract}
Can we evolve better training data for machine learning algorithms? To investigate this question we use population-based optimisation algorithms to generate artificial surrogate training data for naive Bayes for regression. We demonstrate that the generalisation performance of naive Bayes for regression models is enhanced by training them on the artificial data as opposed to the real data. These results are important for two reasons. Firstly, naive Bayes models are simple and interpretable but frequently underperform compared to more complex "black box" models, and therefore new methods of enhancing accuracy are called for. Secondly, the idea of using the real training data indirectly in the construction of the artificial training data, as opposed to directly for model training, is a novel twist on the usual machine learning paradigm.
\end{abstract}

\section{Keywords}

particle swarm optimisation, naive Bayes, regression, artificial data

\section{Introduction}

The typical pipeline for a supervised machine learning project involves firstly the collection of a significant sample of labelled examples typically referred to as training data. Depending on whether the labels are continuous or categorical, the supervised learning task is known as regression or classification respectively. Next, once the training data is sufficiently clean and complete, it is used to directly build a predictive model using the machine learning algorithm of choice. The predictive model is then used to label new unlabelled examples, and if the labels of the new examples are known a priori by the user (but not used by the learning algorithm) then the predictive accuracy of the model can be evaluated. Different models can therefore be directly compared.

In the usual case, the training data is "real", i.e. the model is learned directly from labelled examples that were collected specifically for that purpose. However, quite frequently, modifications are made to the training data after it is collected. For example, it is standard practice to remove outlier examples and normalise numeric values. Moreover, the machine learning algorithm itself may specify modifications to the training data. For example, the widely known bagging algorithm (Breiman, 1996) repeatedly resamples the 
training data with replacement, with each resample being used to train one of multiple models. Similarly the random forest algorithm (Breiman, 2001) repeatedly "projects" the training data in a random fashion in order to produce diverse decision trees. Data augmentation strategies in which artificial data is transformed version of the original data also falls into this category.

In our own previous work (Mayo and Sun, 2014) we introduced the idea of creating entirely new training data for a predictive model using an evolutionary algorithm. In that paper, we performed a very preliminary evaluation in the context of classification. In this paper, we focus specifically on the problem of regression using the naive Bayes for regression (NBR) model (Frank et al, 2000) and perform a much stronger evaluation.

The primary motivation for this line of research is to find methods to improve interpretable machine learning models. Interpretable models are simple models easily understood by users wanting to know, for example, why certain predictions are made, or what knowledge the model has uncovered from the data. A well-known drawback of such models, however, is that that they often lack accuracy to non-interpretable models (e.g. deep neural networks). Can we therefore improve their accuracy somehow?

One approach to solving this problem is to create better interpretable learning algorithms. This is certainly a valid line of inquiry. A second approach, mentioned above, is to preprocess the data somehow to improve the model. How to do that most effectively is also an open question.

The third approach, and the one we focus on here, is to keep the model and its training algorithm, along with the training data, fixed, and instead manipulate the inputs to the learning algorithm. The real training data can be used to measure the quality of the results. In other words, the basic idea is to treat the confluence of the model, its training algorithm, and its training data as a black box and then try to optimise the entire system by treating the inputs to the system (i.e., some training data) as "knobs" that can be tweaked to obtain new behaviours.

An advantage of this approach is that no new algorithms need to be created: instead, existing algorithms and models can be wrapped inside the optimiser and, as we shall see, the potential exists for the models to actually improve in terms of accuracy.

In order to create the artificial training data in an efficient way, the recently proposed competitive swarm optimisation (CSO) algorithm (Cheng and Jin, 2015) is applied. This algorithm optimises a fixed-length numeric vector - a "particle" - such that a given fitness function is minimised. In our approach, particles correspond to artificial training datasets and the fitness function is the error that a NBR model achieves after being trained on the artificial data and tested against the training data.

When the optimisation process is complete, the resulting NBR model - trained on the best artificial dataset - is tested accordingly against held-out test data. We also evaluate an NBR model trained directly on the real training data (i.e., using the "normal" machine learning process) and evaluate it against the same test data.

Our results show that for many benchmark machine learning problems, training an NBR model indirectly on optimised artificial training data significantly improves generalisation performance compared to direct training. Furthermore, the artificial training datasets themselves can be quite small, e.g., containing only three or ten examples, compared to the original training datasets, which may have hundreds or thousands of examples.

The remainder of this paper is structured as follows. In the next section, we briefly survey the two main algorithms used in the research, specifically NBR and CSO. We also briefly touch on previous work in artificial data generation. In the section following that, we describe our new approach more formally, and then we we present results from two rounds of experimentation. The final sections of the paper concern 
firstly an analysis of an artificial dataset produced by our approach on a small $2 \mathrm{D}$ problem, followed by a conclusion.

\section{Background}

\subsection{Naive Bayes for Regression}

Naive Bayes is a popular approach to classification because it is fast and often surprisingly accurate. The naive Bayes approach employs Bayes' rule by assuming conditional independence. Given a set of $m$ categorical random variables $\left\{X_{1}, X_{2}, \ldots, X_{m}\right\}$ and another categorical random variable $Y$ whose value we want to predict, Bayes' rule is given by

$$
\begin{aligned}
& P\left(Y=y \mid X_{1}=x_{1}, X_{2}=x_{2}, \ldots, X_{m}=x_{m}\right)= \\
& \quad P\left(X_{1}=x_{1}, X_{2}=x_{2}, \ldots, X_{m}=x_{m} \mid Y=y\right) P(Y=y) \\
& \sum_{y} P\left(X_{1}=x_{1}, X_{2}=x_{2}, \ldots, X_{m}=x_{m} \mid Y=y\right) P(Y=y)
\end{aligned}
$$

Assuming that the $X_{i}$ are conditionally independent given $Y$, this yields the naive Bayes model:

$$
\begin{aligned}
& P\left(Y=y \mid X_{1}=x_{1}, X_{2}=x_{2}, \ldots, X_{m}=x_{m}\right)= \\
& \frac{\prod_{i=1}^{m} P\left(X_{i}=x_{i} \mid Y=y\right) P(Y=y)}{\sum_{y} \prod_{i=1}^{m} P\left(X_{i}=x_{i} \mid Y=y\right) P(Y=y)}
\end{aligned}
$$

With categorical data, it is trivial to estimate the conditional probabilities $P\left(X_{i}=x_{i} \mid Y=y\right)$. The prior class probability $P(Y=y)$ is based on relative frequencies of corresponding events in the training data. If a predictor variable $X_{i}$ is continuous rather than categorical, it is common to discretise it. Alternatively, one can compute a normal density estimate or a kernel density estimate for $X_{i}$ for each different value of the categorical target variable $Y$.

The assumption of conditional independence is rarely correct in practical applications of this model on real-world data. However, accurate classification does not necessarily require accurate posterior probability estimates $P\left(Y \mid X_{1}, X_{2}, \ldots, X_{m}\right)$ : to achieve high classification accuracy, it is sufficient for the correct class value $Y$ to receive maximum probability; the absolute value of this probability is irrelevant as long as it is larger than the probability for any of the other (incorrect) class values.

In this paper, we consider the NBR algorithm (Frank et al, 2000), which applies to situations with a continuous target variable $Y$. The basic model in the regression case remains the same as the classification case described above. However, estimation of the required conditional densities $f\left(X_{i}=x_{i} \mid Y=y\right)$, as opposed to the conditional probabilities $P\left(X_{i}=x_{i} \mid Y=y\right)$, is more challenging. We provide a brief explanation here. Further details can be found in (Frank et al, 2000).

Assume that $X_{i}$ as well as $Y$ is continuous. We can apply the definition of conditional density to yield

$$
f\left(X_{i}=x_{i} \mid Y=y\right)=\frac{f\left(X_{i}=x_{i}, Y=y\right)}{f(Y=y)}
$$

Both $f\left(X_{i}=x_{i}, Y=y\right)$ and $f(Y=y)$ can then be estimated using a kernel density estimator. In Frank et al (2000), a Gaussian kernel is used in the kernel density estimates and the bandwidths of the kernels are estimated by minimising leave-one-out-cross-validated cross-entropy on a grid of possible bandwidth values. The bandwidths for $X_{i}$ and $Y$ are optimised jointly for $f\left(X_{i}=x_{i}, Y=y\right)$. The obtained bandwidth for $Y$ 
is used to estimate both $f\left(X_{i}=x_{i}, Y=y\right)$ and $f(Y=y)$.

When $X_{i}$ is categorical rather than continuous, we can apply Bayes' rule to obtain

$$
P\left(X_{i}=x_{i} \mid Y=y\right)=\frac{f\left(Y=y \mid X_{i}=x_{i}\right) P\left(X_{i}=x_{i}\right)}{\sum_{x_{i}} f\left(Y=y \mid X_{i}=x_{i}\right) P\left(X_{j}=x_{i}\right)}
$$

Here, $f\left(Y=y \mid X_{i}=x_{i}\right)$ can be estimated using a different kernel density estimator for each value $x_{i}$ of the categorical variable $X_{i}$. The bandwidth for each of these estimators $f\left(Y=y \mid X_{i}=x_{i}\right)$ is again obtained using leave-one-out-cross-validated cross-entropy. The probability $P\left(X_{i}=x_{i}\right)$ can be estimated by counting events in the training set.

The ultimate goal of naive Bayes for regression is prediction based on the posterior density $f\left(Y=y \mid X_{1}=\right.$ $x_{1}, X_{2}=x_{2}, \ldots, X_{m}=x_{m}$ ), which is given by

$$
f\left(Y=y \mid X_{1}=x_{1}, X_{2}=x_{2}, \ldots, X_{m}=x_{m}\right)=\frac{\prod_{i=1}^{m} q\left(X_{i}=x_{i} \mid Y=y\right) f(Y=y)}{\int_{y} \prod_{i=1}^{m} q\left(X_{i}=x_{i} \mid Y=y\right) f(Y=y)}
$$

where

$$
q\left(X_{i}=x_{i} \mid Y=y\right)=\left\{\begin{array}{cc}
P\left(X_{i}=x_{i} \mid Y=y\right) & \text { if } X_{i} \text { is categorical } \\
f\left(X_{i}=x_{i} \mid Y=y\right) & \text { if } X_{i} \text { is continuous }
\end{array}\right.
$$

The integral in the denominator can be obtained using a discrete approximation. In this paper, we use the mode of the density as the predicted value, found using a recursive grid search. Note that the location of the mode can be determined without computing the denominator because its value does not depend on $Y$.

\subsection{Competitive Swarm Optimisation}

Competitive swarm optimisation (CSO) is a recently proposed (Cheng and Jin, 2015) metaheuristic optimisation algorithm derived from particle swarm optimisation (PSO; Kennedy et al, 2001, Bonyadi and Michalewicz, 2017). The PSO family of algorithms are distinguished from other optimisation algorithms by (i) not requiring a gradient function, which enables them to tackle ill-defined problems that are otherwise challenging; and (ii) by performing a goal-directed mutation on solutions (or "particles"), in which several components from the search process (such as various best solutions found so far) contribute to the search procedure.

Similarly to traditional PSO, CSO represents solution vectors as particles. For each particle in CSO, the following information is stored: (i) a position vector $X(t)$ representing a potential solution to the current problem and (ii) a velocity vector $V(t)$ storing $X(t)$ 's trajectory through the solution space. Parameter $t$ is the iteration index and corresponds to time. Both algorithms maintain a single set of particles $P(t)$ comprising $s$ particles in memory. This set is called the swarm.

CSO varies from traditional PSO in several important ways. Firstly, CSO does not maintain a "personal best" memory per particle. Nor does it maintain an explicit "global best" memory for the entire swarm. Instead, the algorithm takes an elitist approach and runs a series of $s / 2$ fitness competitions between random pairs of particles in the swarm. Whichever particle in each competition has the best fitness wins the competition. Winners are not updated for the next iteration. Conversely, the losing particle's velocity and position is updated. Since the global best particle will never lose a fitness competition (at least until it is improved upon), the algorithm will always maintain the global best particle in $P(t)$.

A second key distinction between CSO and PSO is its convergence behaviour. Standard PSO algorithms 
potentially suffer from unbounded particle velocity increases, i.e., particles may travel off "to infinity" along one or more dimensions and therefore the algorithm will not reach an equilibrium. Practical workarounds for this problem are either to limit particle velocities to some maximum magnitude or to carefully select PSO's parameters such that the algorithm converges (Bonyadi and Michalewicz, 2017). CSO requires neither of these workarounds: Cheng and Jin (2015) proved that as long as the key parameter $\varphi$ is non-negative, then the particles will eventually converge on some solution $y^{*}$, which may not be the global optimum but is none-the-less an equilibrium state for the algorithm.

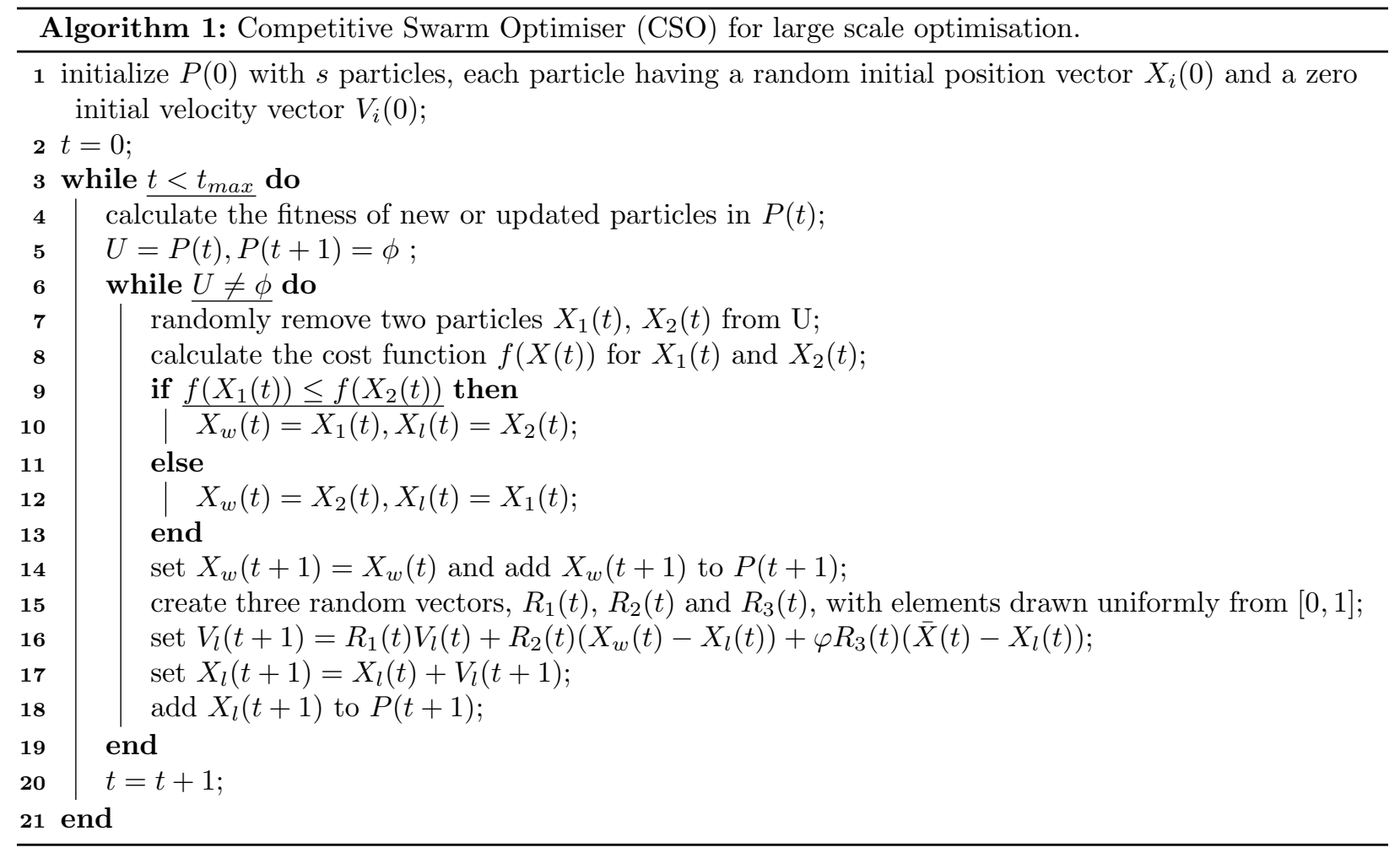

Complete pseudocode for CSO, adapted from Cheng and Jin (2015), is given in Algorithm 1. The pseudocode assumes that a fitness function $f$ over particles, to be minimised, is provided. Essentially, CSO consists of two loops: an outer loop over iterations defined at line 3 , and an inner loop over fitness competitions per iteration defined at line 6. The fitness values are computed on line 8 , Line 14 shows how the winner of the current competition passes through to the next iteration unchanged.

The rules for updating the velocity and position of each losing particle are specified in lines 15 17. The first of these lines creates three random vectors. These are used to mix three different components together in order to compute a new velocity for the particle being updated. The three components are (i) the particle's original velocity which provides an element of inertia to the velocity updates; (ii) the difference between the particle's current position and the position of the particle that it just lost to; and (iii) the difference between the particle and the swarm mean $\bar{X}(t)$. The updated velocity is then added to the losing particle's position so that the particle moves to a new position in search space.

A key aspect of the algorithm is that the influence of the swarm mean is controlled by the parameter $\varphi$. When $\varphi=0$, the swarm mean does not need to be computed and is effectively ignored; when $\varphi>0$, however, the swarm mean has an increasingly attractive effect on the particles. Overall, the algorithm has 
fewer parameters than other similar algorithms. Besides $\varphi$, the only other critical parameters are $s$, the number of particles in the swarm, and $t_{\max }$, the number of iterations to perform. In the general case, $t_{\max }$ can be changed to an alternative stopping criterion.

Prior evaluations (Cheng and Jin, 2015) have shown that CSO outperforms other population-based metaheuristic algorithms on standard high-dimensional benchmark optimisation functions. Therefore, due to its simplicity and excellent performance, we adopt CSO for this research.

\subsection{Prior Work on Artificial Example Generation}

The creation of artificial training data for testing new machine learning algorithms (as opposed to training the models) has a long history. The basic idea is to create training data that has known patterns or properties, and then observe the algorithm's performance. For example, does the algorithm correctly detect known patterns that exist in the artificial data? Answering these questions can lead to insight about how well the algorithm will perform in with real datasets. A well-known example of an artificial dataset generator is the "people" data generator described by Agrawal et al (1993).

Another class of artificial training data generation is used for speeding up nearest neighbour classifiers. Nearest neighbour classification can be computationally expensive if the number and/or dimensionality of the examples is high. A suite of algorithms for optimising the set of possible neighbours therefore exists in the literature: see Triguero et al (2011) for a comprehensive survey. In most cases these algorithms focus on example selection from the real data rather than the generation of new examples. However, algorithms have been proposed for both modifying existing examples (e.g., via compression as performed by Zhong et al (2017)) and also for creating entirely new examples, such as in the work by Impedovo et al (2014) which describes a method for digit prototype generation. Escalante et al (2013) also describe a method of example generation in which genetic programming is applied.

Artificial data generation techniques can be used to address the class imbalance problem. Many machine learning algorithms for classification fail to work effectively when the classes are severely imbalanced. In the classic machine learning literature, Chawla et al (2002) developed the synthetic minority over-sampling technique (SMOTE) to artificially increase the size of the minority class, (producing better class balance in the data) while correspondingly preserving the examples in the majority class. Similarly, Lòpez et al (2014) developed a new technique based on evolutionary algorithms for generating minority class examples.

It is worth mentioning here the significant number of works in the literature showing that corrupting training data with noise improves the resulting generalisation performance of models. This idea is common in the neural network literature. For example, Guozhong (1996) found that input data corruption improved neural network generalisation for both classification and regression tasks. More recently, stacked denoising autoencoders (Vincent et al, 2008), which are based on the idea of corrupting inputs to learn more robust features in deep networks, have seen considerable success. Likewise, in the evolutionary machine learning literature, dataset corruption has been demonstrated to be an effective strategy for learning both classifier systems (Urbanowicz et al, 2011) and symbolic discriminant analysis models (Greene et al, 2009). These approaches do not strictly generate artificial examples; rather they are based on perturbing the real training data.

Finally, we briefly mention generative adversarial nets (GANs) proposed by Goodfellow et al (2014). These are a type of paired neural network in which one network "generates" artificial data examples by passing random noise through the network, and the other network "discriminates" between the examples in order to to determine if the examples are from the real training dataset or are fakes. The purpose of this 
process is to construct better generative models from the data.

In contrast, the work we present in this paper extends and build on work originally reported in Mayo and Sun (2014). Rather than aiming to generate fake data that is indistinguishable from real data, we aim to produce artificial data maximising the performance of the resulting classifier when it is trained on the artificial data. Therefore, the artificial data may (or may not) be easily distinguishable from the real data. In fact, in some cases the artificial data is easily distinguishable from the real data. For example, an artificial dataset depicted discussed shortly has negative values for the prediction target: such values do not occur in the corresponding real data. The approach proposed in this paper is therefore quite different in nature to the GAN approach.

To summarise, prior work has explored many variations on the theme of perturbing the training data in different ways in order to improve model performance, or focussed on example generation strategies for specific purposes (such as balancing the data). Mayo and Sun (2014) is the only prior work investigating the generation of completely new artificial training datasets. In that work, naive Bayes for classification was shown to perform well with the optimised artificial data. In this paper, we significantly extend that work by (i) applying the same basic approach to naive Bayes for regression; (ii) updating the metaheuristic algorithm used for the optimisation to CSO, a more modern algorithm designed specifically for high dimensional problems; and (iii) testing against a wide range of varied regression problems.

\section{Evolving Artificial Data for Regression}

In this section, we precisely outline our approach to constructing artificial training data for regression problems using CSO.

The classical approach to machine learning is to start with some tabular training data $D_{\text {train }}$, comprising $r$ rows or examples, and $d$ attributes. One of the attributes, typically the first or last one, is the target to predict and the other attributes are predictor variables.

Given this training data, we can think of a machine learning algorithm such as NBR as a function of the training data, i.e.,

$$
m_{1}=m_{N B R}\left(D_{\text {train }}\right)
$$

where $m_{1}$ is a predictive model and $m_{N B R}$ is a function encapsulating a machine learning algorithm that trains an NBR model given some training dataset. The performance of the model on a test dataset can similarly be stated as a function

$$
e_{1}=\operatorname{eval}\left(m_{1}, D_{\text {test }}\right)
$$

with $e_{1}$ being a standard metric for regression error such as root mean squared error (RMSE).

Our approach is based on defining a corresponding fitness function $f(X(t))$ for CSO. In order to evaluate $f(X(t))$, we must first convert particle $X(t)$ into a tabular dataset with $n$ examples where $n$ is small and fixed. This we will refer to as $D_{X(t)}$. Typically we will have $n \ll r$ unless $r$ itself is very small. Since the entire dataset $D_{X(t)}$ has $d n$ unique values, then the dimensionality of the particles $X(t)$ is $d n$.

The process of converting $X(t)$ to $D_{X(t)}$ is fairly straightforward: for numeric attributes, the values are copied directly from the particle into the correct position in $D_{X(t)}$ without modification. For categorical (i.e., discrete) attributes, the process is complicated by the fact that CSO optimises continuous values only. We correct for this by rounding individual values from $X(t)$ to the nearest legal discrete value when they are copied across to $D_{X(t)}$. The original values in $X(t)$ remain unmodified. 
Random particle initialisation (line 1 of Algorithm 1), is performed by an examination of $D_{\text {train }}$ : each variable is initialised to a random uniform value between the minimum and maximum values of the corresponding attribute in $D_{\text {train }}$.

Thus, we can define the fitness function to minimise as the error some model $m_{2}$ yields on the training data:

$$
f(X(t))=\operatorname{eval}\left(m_{2}, D_{\text {train }}\right)
$$

where $m_{2}$ is not trained on the original training data, but instead on the artificial dataset that is derived from the current particle $X(t)$ being evaluated, i.e.

$$
m_{2}=m_{N B R}(g(X(t)))=m_{N B R}\left(D_{X(t)}\right)
$$

where the function $g$ converts the one-dimensional particle $X(t)$ into a tabular dataset $D_{X(t)}$ using the procedure outlined above.

After the optimisation process is complete, we can evaluate $m_{2}$ against the test data to get the test error

$$
e_{2}=\operatorname{eval}\left(m_{2}, D_{\text {test }}\right)
$$

This will enable us to perform a direct comparison with $e_{1}$, the test error achieved using the traditional machine learning approach.

In terms of computational time complexity, the significant cost of this algorithm is the fitness function evaluation. Evaluating the fitness of a particle involves firstly converting the particle to a dataset; secondly, training a regression model using the dataset; and thirdly, evaluating that model against the real dataset. The training and testing steps clearly have the most significant complexity in this operation. Since CSO needs at least a few hundred iterations to find good solutions, this implies that our approach may not be feasible with models that are very time consuming to train or test, which may be due to either the specific learning algorithm's own complexity or the real dataset being very large in size. Measures may therefore need to be taken (e.g. replacing the algorithm with a more efficient one, or only using a subset of the real data for fitness evaluations) in these cases.

As mentioned, we have chosen a direct representation in which variables being optimised map directly to attribute values in the artificial dataset. A consequence of this is that different particles (the equivalent of "genotypes" in evolutionary computing) may result in identical models (i.e., the equivalent of "phenotypes") if the learning algorithm ignores the order of the examples in the data. For example, the datasets $\left\{x_{1}, x_{2}, x_{3}\right\}$ and $\left\{x_{2}, x_{3}, x_{1}\right\}$, where $x_{i}$ is an example, produce the same NBR model. This observation is not necessarily a drawback of our approach. In fact, in many other approaches, multiple genotypes mapping onto a single phenotype is a deliberate feature as it enables neutral mutations. For example, linear genetic programming (LGP), proposed by Brameier and Banzhaf (2007), evolves sequential programs. Since the order of any two instructions is often not dependent (e.g., initialising two different variables), the same issue arises: different programs map to the same behaviours. However, this does suggest that future research could investigate alternative particle/dataset mapping functions to determine if efficiencies are possible over the approach we use here. 
Table 1: Dataset characteristics used in the experiments. The third column shows the total number of features, with the total number of numeric features in parentheses.

\begin{tabular}{|c|c|c|}
\hline Dataset & \#Examples & \#Features \\
\hline auto93 & 93 & $22(16)$ \\
\hline autoHorse & 205 & $25(17)$ \\
\hline autoMpg & 398 & $7(4)$ \\
\hline autoPrice & 159 & $15(15)$ \\
\hline baskball & 96 & $4(4)$ \\
\hline bodyfat & 252 & $14(14)$ \\
\hline bolts & 40 & $7(7)$ \\
\hline cholesterol & 303 & $13(6)$ \\
\hline cleveland & 303 & $13(6)$ \\
\hline cloud & 108 & $6(4)$ \\
\hline cpu & 209 & $7(6)$ \\
\hline detroit & 13 & $13(13)$ \\
\hline echoMonths & 130 & $9(6)$ \\
\hline elusage & 55 & $2(1)$ \\
\hline fishcatch & 158 & $7(5)$ \\
\hline fruitfly & 125 & $4(2)$ \\
\hline gascons & 27 & $4(4)$ \\
\hline housing & 506 & $13(12)$ \\
\hline hungarian & 294 & $13(6)$ \\
\hline longley & 16 & $6(6)$ \\
\hline lowbwt & 189 & $9(2)$ \\
\hline mbagrade & 61 & $2(1)$ \\
\hline meta & 528 & 21(19) \\
\hline $\mathrm{pbc}$ & 418 & $18(10)$ \\
\hline pharynx & 195 & $11(1)$ \\
\hline pollution & 60 & $15(15)$ \\
\hline pwLinear & 200 & $10(10)$ \\
\hline quake & 2178 & $3(3)$ \\
\hline schlvote & 38 & $5(4)$ \\
\hline sensory & 576 & $11(0)$ \\
\hline servo & 167 & $4(0)$ \\
\hline sleep & 62 & $7(7)$ \\
\hline strike & 625 & $6(5)$ \\
\hline veteran & 137 & $7(3)$ \\
\hline vineyard & 52 & $3(3)$ \\
\hline
\end{tabular}

\section{Initial Evaluation}

We conducted an initial evaluation of our proposed algorithm on benchmark regression datasets used in Frank et al (2000) 1 Although small, these datasets do represent a diverse range of prediction problems and have been acquired from several different sources.

To illustrate the diversity, the "fruit fly" dataset consists of observations describing male fruit fly sexual behaviour and is used to build a model predicting fly longevity. Conversely, the "cpu" dataset is used to construct a predictive model for CPU performance given various technical features of a CPU. Finally, the "pollution" dataset is used to predict the age-adjusted mortality rate of a neighbourhood given various metrics such as the amount of rainfall and the degree of presence of various pollutants. Overall, there are 35 distinct regression datasets in the collection, with between 38 to 2178 examples per dataset. The dimensionality of the datasets ranges from 2 to 25 . Table 1 gives the exact properties of each dataset.

\footnotetext{
${ }^{1}$ Original datasets available from http://prdownloads.sourceforge.net/weka/datasets-numeric.jar
} 
Certainly, the datasets themselves are not "high dimensional" datasets by any standard. However, when the proposed optimisation algorithm is applied to evolve artificial datasets with, e.g., ten examples, then the dimensionality for the optimisation problem ranges between 30 and 260 depending on the dataset. Thus, the dimensionality of the optimisation problem high-dimensional.

To prepare the datasets for our evaluation, we split each dataset into a training portion (consisting of a randomly selected $66 \%$ of the examples) and a held-out test portion (consisting of the remaining examples). Maintaining a fixed test set was necessary so that the variability in the results produced by our stochastic search method could be assessed properly.

The key parameters for CSO, specifically $\varphi, t_{\max }$ and $s$ were set of $0.1,1000$, and 100 respectively. For evaluating the models, the fitness function to be minimised was RMSE, and we fixed the size of the artificial datasets to $n=10$. These initial parameter values were chosen after some initial exploratory tests on one of the datasets.

Because CSO is a heavily randomised search algorithm and will therefore give different results each time it is run, we ran our algorithm ten times for on each train/test split. Thus, we executed a total of 350 runs of our algorithm overall.

Table 2 gives the results by dataset. The column "NBR" in the table gives the baseline testing performance of NBR trained using the traditional process. We further include in the table the results achieved using linear regression (LR) and Gaussian process regression with radial basis function kernels (GPR) as implemented in WEKA 3.8.0 (Frank et al, 2016). The implementation of LR performs attribute selection using the M5' method (Wang and Witten, 1997), which usually outperforms standard LR. We tuned the kernel parameter and the noise parameter of GPR using a grid search that optimised parameters based on internal cross-validation of the root mean squared error on the training set. We used the range $10^{0} \ldots 10^{-5}$ for the noise parameter and the range $10^{5} \ldots 10^{-5}$ for the kernel parameter. Note that GPR is a state-of-the-art black box regression method and therefore it is expected that it will outperform methods that produce simple models.

The error metrics on the test splits achieved by our experimental algorithm are given in the columns "CSO-NBR(mean)" and "CSO-NBR(best)", which give the average ( \pm the standard deviation) and best errors achieved respectively over the ten repetitions.

Finally, we also performed a single-sample, one-sided $t$-test comparing the results of our experimental algorithm with the single result achieved by traditional NBR. The $p$ value for the test is given as well in Table 2 .

Overall, we can see NBR accuracy performance is improved significantly in most cases when CSO-NBR is used to train the model. If we consider the strong condition that $p<0.01$ (i.e., $99 \%$ confidence), then NBR's performance is improved in $18 / 35$ cases. If the condition is weakened to $p<0.05$ (95\% confidence), then a further two more significant improvements can be added to that total.

Also interesting is the comparison of NBR, LR, and our experimental algorithm. NBR in its original form tends to underperform LR on most of the problems; in fact, on 25/35 LR has a lower error than NBR. However, when we compare the mean performance of CSO-NBR to LR, the number of wins for LR decreases to $21 / 35$. If we furthermore consider the error of the best single run of our experimental algorithm, then the number of LR wins drops to only $8 / 35$ datasets.

We note that considering the best-of-ten-runs result (i.e., the "CSO-NBR(best)" column) is an optimistic strategy, and it has consequently been de-emphasized in Table 2 However, it is worth including these results because in practice, if only one model is required but CSO-NBR is run multiple times (which it should be), 
Table 2: Initial experiment results on the small datasets. Shown are the test errors. To enhance readability, an asterisk $\left({ }^{*}\right)$ indicates the best result in an LR vs. NBR vs. CSO-NBR(mean) comparison, while bold indicates the best result in a NBR vs. CSO-NBR(mean) comparison. We also include the results achived by parameter-optimised GPR as a reference.

\begin{tabular}{|c|c|c|c|c|c|c|}
\hline Dataset & GPR & LR & NBR & CSO-NBR(mean) & $p$ value & CSO-NBR(best) \\
\hline auto93 & 5.00 & 11.08 & ${ }^{*} 5.41$ & $5.91 \pm 1.89$ & 0.7880 & 3.92 \\
\hline autoHorse & 11.19 & 13.87 & *12.92 & $13.00 \pm 2.59$ & 0.5401 & 9.26 \\
\hline autoMpg & 3.02 & $* 3.15$ & 3.43 & $3.55 \pm 0.12$ & 0.9926 & 3.32 \\
\hline autoPrice & 2503.06 & 2640.96 & 2375.45 & ${ }^{*} 2261.03 \pm 218.19$ & 0.0658 & 1894.58 \\
\hline baskball & 0.08 & ${ }^{*} 0.08$ & 0.11 & $0.09 \pm 0.01$ & 0.0007 & 0.08 \\
\hline bodyfat & 0.64 & 0.72 & 1.68 & ${ }^{*} 0.53 \pm 0.09$ & 0.0000 & 0.41 \\
\hline bolts & 13.93 & 11.29 & ${ }^{*} 7.92$ & $11.46 \pm 8.35$ & 0.8938 & 7.52 \\
\hline cholesterol & 51.17 & *50.95 & 61.76 & $56.92 \pm 3.81$ & 0.0015 & 51.63 \\
\hline cleveland & 0.77 & ${ }^{*} 0.79$ & 1.05 & $0.80 \pm 0.04$ & 0.0000 & 0.74 \\
\hline cloud & 0.32 & $* 0.31$ & 0.43 & $0.37 \pm 0.12$ & 0.0881 & 0.31 \\
\hline $\mathrm{cpu}$ & 31.68 & 93.75 & 114.65 & ${ }^{*} 43.70 \pm 6.85$ & 0.0000 & 28.37 \\
\hline detroit & 27.8 & * 48.24 & 89.18 & $52.24 \pm 20.06$ & 0.0001 & 21.79 \\
\hline echoMonths & 11.22 & * 11.21 & 12.67 & $12.89 \pm 1.70$ & 0.6526 & 11.32 \\
\hline elusage & 14.81 & ${ }^{*} 12.94$ & 17.73 & $17.52 \pm 1.26$ & 0.3002 & 16.12 \\
\hline fishcatch & 61.61 & 104.76 & 209.45 & ${ }^{*} 63.68 \pm 18.68$ & 0.0000 & 45.83 \\
\hline fruitfly & 14.94 & ${ }^{*} 14.32$ & 19.80 & $16.94 \pm 0.70$ & 0.0000 & 15.67 \\
\hline gascons & 11.18 & 40.27 & 17.63 & ${ }^{*} 3.23 \pm 0.76$ & 0.0000 & 2.17 \\
\hline housing & 3.64 & 5.20 & 6.32 & ${ }^{*} 3.92 \pm 0.14$ & 0.0000 & 3.70 \\
\hline hungarian & 0.31 & $* 0.30$ & 0.36 & $0.33 \pm 0.02$ & 0.0001 & 0.30 \\
\hline longley & 425.42 & 803.03 & ${ }^{*} 552.15$ & $755.90 \pm 428.00$ & 0.9168 & 282.72 \\
\hline lowbwt & 491.03 & *496.61 & 559.99 & $550.26 \pm 47.09$ & 0.2649 & 501.34 \\
\hline mbagrade & 0.30 & ${ }^{*} 0.30$ & ${ }^{*} 0.30$ & $0.34 \pm 0.02$ & 1.0000 & 0.33 \\
\hline meta & 321.00 & 398.92 & 378.52 & ${ }^{*} 365.57 \pm 121.54$ & 0.3719 & 217.87 \\
\hline $\mathrm{pbc}$ & 947.50 & *970.26 & 1049.74 & $1016.99 \pm 49.78$ & 0.0336 & 922.27 \\
\hline pharynx & 288.27 & 429.51 & 354.80 & ${ }^{*} 327.16 \pm 15.86$ & 0.0002 & 301.58 \\
\hline pollution & 46.36 & *46.29 & 62.31 & $55.84 \pm 8.78$ & 0.0224 & 42.64 \\
\hline pwLinear & 1.85 & 2.45 & 2.49 & ${ }^{*} 2.04 \pm 0.05$ & 0.0000 & 1.96 \\
\hline quake & 0.20 & 0.20 & 0.27 & ${ }^{*} 0.19 \pm 0.00$ & 0.0000 & 0.19 \\
\hline schlvote & 1855748.32 & 1795017.06 & 1956688.47 & ${ }^{*} 1096037.48 \pm 67480.04$ & 0.0000 & 1023308.85 \\
\hline sensory & 0.75 & ${ }^{*} 0.79$ & 0.94 & $0.82 \pm 0.02$ & 0.0000 & 0.79 \\
\hline servo & 0.74 & ${ }^{*} 0.84$ & 1.04 & $0.88 \pm 0.05$ & 0.0000 & 0.82 \\
\hline sleep & 2.77 & ${ }^{*} 2.79$ & 4.09 & $3.49 \pm 0.55$ & 0.0038 & 2.81 \\
\hline strike & 657.92 & *662.13 & 696.60 & $680.04 \pm 29.17$ & 0.0531 & 663.30 \\
\hline veteran & 157.83 & * 155.44 & 164.17 & $181.64 \pm 31.27$ & 0.9444 & 155.39 \\
\hline vineyard & 2.52 & 1.80 & ${ }^{*} 1.33$ & $2.72 \pm 0.56$ & 1.0000 & 2.16 \\
\hline
\end{tabular}


then only the best single model during testing is likely to be used for subsequent analysis (e.g., for inspecting model, or for use in live production).

In terms of individual dataset performance, the difference between NBR and CSO-NBR is quite variable. For example, the "gascons" dataset appears to be very difficult to model for both NBR and LR, which achieve test set errors of 17.63 and 40.27 respectively. CSO-NBR, on the other hand, generalises quite well, achieving a mean testing error of only 3.23. A similar example is the "bodyfat" problem, in which test errors of 1.68 and 0.72 for NBR and LR respectively can be compared to the 0.53 mean test error that CSO-NBR achieves.

Conversely, there are also datasets where CSO-NBR performs extremely poorly: for example, the "longley" dataset is modelled well by NBR but poorly by both LR and CSO-NBR. Similarly for the "vineyard" dataset. There appears to be no clear-cut single best algorithm overall.

Also interesting is a pattern that is clearly evident in the table of results. If one considers the cases where CSO-NBR is statistically significantly better than NBR, it appears to also be the case (in all but one instance) that LR also shows a large reduction in error compared to NBR. This suggests that those datasets have properties that NBR cannot model well by itself, without being embedded inside CSO. This certainly warrants future investigation, perhaps in the context of using synthetic data to determine which patterns NBR and CSO-NBR can and cannot detect.

In terms of a comparison between the methods that produce simple and interpretable models and the parameter-optimised black box method GPR, as expected, GPR achieves significant performance improvements on average. However, in five cases (namely "autoPrice", "bodyfat", "gascons", "quake" and "schlvote"), CSO-NBR outperforms GBR, sometimes by a wide margin.

Finally, we were also interested in a comparison of CSO to a more traditional PSO variant. This was to determine if CSO was really a suitable optimiser for this problem. To that end, we selected the standard PSO algorithm (SPSO) described by Bonyadi and Michalewicz (2017). SPSO has three main parameters: an inertia parameter, a cognitive constant, and a social constant. Following the recommendation by Bonyadi and Michalewicz, 2017, pp. 11) we set these parameters to 0.6, 1.7 and 1.7 respectively.

A fundamental difference between the CSO and SPSO is that SPSO updates all particles in the swarm at each iteration whereas CSO updates only half of the particles (the losers) per iteration. Similarly, particles in CSO have no personal memory; particles in SPSO on the other hand each have a memory for their "personal best" position additional to their current position. Therefore it is unfair to directly compare SPSO and CSO with the same swarm size and same number of iterations, as SPSO would be performing approximately twice the number of function evaluations, and furthermore, it would use twice the amount of memory as CSO.

To make comparisons fairer, we gave each algorithm approximately the same number of fitness function evaluations, since the fitness function is the main contributor to the algorithm complexity. There are two ways of making the number of fitness evaluations performed by SPSO approximately equal to those performed by CSO: halving the swarm size or halving the number of iterations. We tested both variants, i.e., we defined one version of SPSO-NBR with a $s=50$ and $t_{\max }=1000$, and another with $s=100$ and $t_{\max }=500$. The latter version of SPSO uses the twice the amount of memory as the other two algorithms.

The SPSO results are given in the tables in the appendix. A comparison of these tables with the CSO-NBR results in Table 2 show that in most cases, CSO-NBR outperforms SPSO-NBR. Specifically, the $s=50, t_{\max }=1000$ variant of SPSO-NBR outperforms CSO-NBR on only six of the datasets while the $s=100, t_{\max }=500$ variant (using twice as much memory) outperforms CSO-NBR on twelve of the datasets.

We continue in the next section using CSO only, as (i) it has been shown that CSO generally outperforms 
Table 3: Datasets used in the experiment.

\begin{tabular}{|c|c|c|}
\hline Dataset & \#Examples & \#Features \\
\hline Wine Quality - Red $(\overline{\text { Cortez et al }} \mid \overline{2009})$ & 1,599 & 11 \\
\hline Wine Quality - White (Cortez et al, 2009) & 4,898 & 11 \\
\hline Energy Efficiency - Heating (Tsanas and Xifara, 2012) & 768 & 8 \\
\hline Energy Efficiency - Cooling ( Tsanas and Xifara $\overline{2012}$ ) & 768 & 8 \\
\hline Parkinson's - Motor (Tsanas et al 2010$)$ & 5,875 & 18 \\
\hline Parkinson's - Total (Tsanas et al, 2010$)$ & 5,875 & 18 \\
\hline Appliance Energy (Candanedo et al 2017) & 19,373 & 26 \\
\hline Survival (Lenz et al, 2008 Wang, 2015) & 181 (train)/233 (test) & 3,833 \\
\hline
\end{tabular}

SPSO on the same problems, or is at least in the same ballpark; and (ii) CSO requires less parameter tuning compared to SPSO. To summarise, our initial experiments indicate that our experimental algorithm - training an NBR model on artificial training data optimised in a supervised fashion using CSO - is an effective approach for constructing more robust naive Bayes regression models for certain machine learning problems. We therefore proceed to a more challenging set of problems in the next round of experiments.

\section{Parameter Study with Modern Datasets}

In this set of experimental runs, we consider the effect of various key parameters on the artificial data optimisation process, in particular CSO's $\varphi$ parameter, and $n$, the size of the artificial dataset.

We ran experiments with $\varphi \in\{0.0,0.1,0.5,1.0\}$ and $n \in\{3,10,20\}$ to evaluate the effect on the generalisation error of the resulting NBR model. The remaining parameters, specifically $s$, the swarm size, and $t_{\max }$, the number of iterations, were held at constant values (100 and 1000 respectively) so that the total number of function evaluations was a constant. Recall that each function evaluation consists of one run of NBR on the artificial data to train the model followed by a testing procedure to measure the RMSE on the real training data.

We chose eight new regression problems for this round of experiments. These datasets are larger than those used in the previous round of experiments, and also much more recently published. Table 3 summarises the datasets, giving their name and the overall dimensionality of the data in terms of number of examples and number of predictive features excluding the target value.

In brief, the wine quality datasets (Cortez et al, 2009) contains data about various Portuguese "Vinho Verde" wines. The features are physiochemical statistics about each wine (e.g., the pH value) and the target to predict is the subjective quality of the wine, which ranges on a scale from 0 to 10 . The data is divided into two separate prediction problems, one for red wine and one for white wine with the white wine dataset being considerably larger in size.

The building energy efficiency datasets (Tsanas and Xifara, 2012), in contrast, concerns predicting the heating and/or cooling load of a building, given various properties of the building's design. The buildings are simulated, and while each building has the same volume, the surface areas, dimensions, and the materials used to construct the buildings vary. There are eight predictive features in total, and for each example there are two separate prediction targets: (i) the total heating load and (ii) the total cooling load. Accurately predicting the loads of the buildings without running full (and highly computationally complex) building simulations is an important problem in the optimisation of energy efficient buildings.

The fifth and sixth problems deal with the remote diagnosis of Parkinson's disease (Tsanas et al, 2010). The aim of this data is to learn a model that can predict the Unified Parkinson's Disease Rating Scale 
Table 4: Experiment results (test errors) on the Wine Quality - Red dataset.

\begin{tabular}{lrrrr}
\hline$\varphi$ & $n$ & NBR & CSO-NBR(mean) & CSO-NBR(best) \\
\hline 0.0 & 3 & 0.8522 & $0.6735 \pm 0.0037$ & 0.6670 \\
0.0 & 10 & 0.8522 & $0.6745 \pm 0.0079$ & 0.6651 \\
0.0 & 20 & 0.8522 & $0.6885 \pm 0.0164$ & 0.6656 \\
0.1 & 3 & 0.8522 & $0.6703 \pm 0.0066$ & 0.6613 \\
0.1 & 10 & 0.8522 & $0.6751 \pm 0.0098$ & 0.6591 \\
0.1 & 20 & 0.8522 & $0.6756 \pm 0.0072$ & 0.6630 \\
0.5 & 3 & 0.8522 & $0.6757 \pm 0.0066$ & 0.6641 \\
0.5 & 10 & 0.8522 & $0.6668 \pm 0.0090$ & 0.6543 \\
0.5 & 20 & 0.8522 & $0.6711 \pm 0.0072$ & 0.6579 \\
1.0 & 3 & 0.8522 & $0.6775 \pm 0.0090$ & 0.6620 \\
1.0 & 10 & 0.8522 & $0.6705 \pm 0.0122$ & 0.6534 \\
1.0 & 20 & 0.8522 & $\mathbf{0 . 6 6 6 4} \pm \mathbf{0 . 0 0 9 9}$ & $\mathbf{0 . 6 5 1 9}$ \\
\hline
\end{tabular}

(UPDRS) for a patient. Normally, the UPDRS score is determined by trained medical personnel in the clinic using a costly physical exam. In contrast, the dataset constructed for this problem uses statistical features extracted via the signal processing of recorded speech alone. Thus, UPDRS assessment can be performed remotely instead of in-person if the predictions are accurate enough. Similar to the previous problem, this prediction problem has two predictive targets per example: the total UPDRS score, ranging from 0 (healthy) to 176 (total disability); and the motor UPDRS score, which has a smaller range of 0 to 108.

The seventh problem, like the building energy prediction problems, is concerned with energy usage (Candanedo et al, 2017). Unlike those problems, however, this dataset is derived from real data rather than a simulation and is much larger: there are nearly twenty thousand examples in total, and the dimensionality is higher as well. The problem is to predict the energy usage of appliances in a real house given features related to the environment, such as weather data and temperature/humidity readings from wireless sensors. The data is arranged into a time series with each example corresponding to data about the weather and household/appliance usage activity over a ten minute period.

Finally, the eighth dataset we considered concerns building a regression model to predict survival time for cancer patients given gene expression data. The data was originally acquired by Lenz et al (2008) and subsequently further processed and re-published by Wang (2015). The features in the dataset are measurements from probe sets obtained using a microarray device. Each measurement is an estimate of one particular gene's degree of expression in a patient. The prediction target is the survival time of the patient. Because of the high dimensionality of the data, we performed our experiments on this data using a random subset of one hundred of the gene expressions measurements. Such an approach is commonly used elsewhere. For example, Kapur et al (2016) used the same strategy when learning Bayesian network models for gene expression class prediction.

In terms of the experimental setup, our experiments followed the same basic procedure that we applied previously: i.e., the data was split into training and test portions, and then both standard NBR and LR served as baselines. We ran CSO-NBR ten times to assess its average and best performance on each problem.

With respect to splitting the data into training and test subsets, for the first six datasets, the ordering of the examples was randomised and the split sizes were two-thirds and one-third for the training and test sets respectively. The Appliance Energy data, however, forms a time series. Therefore we did not randomise the examples but instead preserved their order, which resulted in the training examples preceding the test examples in time. The split sizes were the same as before however. For the Survival data, the examples are already arranged into training and test subsets by Wang (2015), and we did not alter this. 
Table 5: Experiment results (test errors) on the Wine Quality - White dataset.

\begin{tabular}{lrrrr}
\hline$\varphi$ & $n$ & NBR & CSO-NBR(mean) & CSO-NBR(best) \\
\hline 0.0 & 3 & 1.2724 & $0.8270 \pm 0.0095$ & 0.8107 \\
0.0 & 10 & 1.2724 & $0.8285 \pm 0.0101$ & 0.8144 \\
0.0 & 20 & 1.2724 & $0.8624 \pm 0.0734$ & 0.8266 \\
0.1 & 3 & 1.2724 & $0.8272 \pm 0.0105$ & 0.8138 \\
0.1 & 10 & 1.2724 & $0.8202 \pm 0.0069$ & 0.8133 \\
0.1 & 20 & 1.2724 & $\mathbf{0 . 8 2 0 0} \pm \mathbf{0 . 0 1 1 3}$ & $\mathbf{0 . 8 0 4 2}$ \\
0.5 & 3 & 1.2724 & $0.8319 \pm 0.0098$ & 0.8171 \\
0.5 & 10 & 1.2724 & $0.8285 \pm 0.0068$ & 0.8202 \\
0.5 & 20 & 1.2724 & $0.8246 \pm 0.0093$ & 0.8134 \\
1.0 & 3 & 1.2724 & $0.8361 \pm 0.0094$ & 0.8210 \\
1.0 & 10 & 1.2724 & $0.8256 \pm 0.0083$ & 0.8109 \\
1.0 & 20 & 1.2724 & $0.8237 \pm 0.0056$ & 0.8152 \\
\hline
\end{tabular}

Table 6: Experiment results (test errors) on the Energy Efficiency - Heating dataset.

\begin{tabular}{lrrrr}
$\varphi$ & $n$ & NBR & CSO-NBR(mean) & CSO-NBR(best) \\
\hline 0.0 & 3 & 6.2906 & $3.9545 \pm 0.4241$ & 3.4115 \\
0.0 & 10 & 6.2906 & $4.4217 \pm 0.8192$ & 3.1010 \\
0.0 & 20 & 6.2906 & $4.9732 \pm 0.9185$ & 3.5728 \\
0.1 & 3 & 6.2906 & $\mathbf{3 . 7 8 4 9} \pm \mathbf{0 . 6 2 1 1}$ & $\mathbf{2 . 5 7 5 2}$ \\
0.1 & 10 & 6.2906 & $3.9914 \pm 0.4370$ & 2.9801 \\
0.1 & 20 & 6.2906 & $4.5730 \pm 1.6502$ & 2.8295 \\
0.5 & 3 & 6.2906 & $4.3207 \pm 0.8469$ & 3.5456 \\
0.5 & 10 & 6.2906 & $4.6647 \pm 0.4799$ & 3.6592 \\
0.5 & 20 & 6.2906 & $4.4657 \pm 1.0296$ & 2.9383 \\
1.0 & 3 & 6.2906 & $4.0320 \pm 0.5351$ & 3.3858 \\
1.0 & 10 & 6.2906 & $5.0931 \pm 0.9932$ & 3.5922 \\
1.0 & 20 & 6.2906 & $4.7041 \pm 0.6888$ & 3.8492 \\
\hline
\end{tabular}

Table 7: Experiment results (test errors) on the Energy Efficiency - Cooling dataset.

\begin{tabular}{lrrrr}
\hline$\varphi$ & $n$ & NBR & CSO-NBR(mean) & CSO-NBR(best) \\
\hline 0.0 & 3 & 5.5104 & $3.5554 \pm 0.4713$ & 2.8654 \\
0.0 & 10 & 5.5104 & $4.0276 \pm 0.5111$ & 3.3790 \\
0.0 & 20 & 5.5104 & $4.9107 \pm 0.4092$ & 4.1077 \\
0.1 & 3 & 5.5104 & $3.5219 \pm 0.3873$ & 2.8390 \\
0.1 & 10 & 5.5104 & $\mathbf{3 . 2 0 1 5} \pm \mathbf{0 . 3 9 1 6}$ & $\mathbf{2 . 5 1 2 8}$ \\
0.1 & 20 & 5.5104 & $4.4863 \pm 2.4956$ & 2.9844 \\
0.5 & 3 & 5.5104 & $3.4576 \pm 0.3586$ & 2.9568 \\
0.5 & 10 & 5.5104 & $3.4896 \pm 0.5219$ & 2.5689 \\
0.5 & 20 & 5.5104 & $4.0933 \pm 0.8163$ & 3.1503 \\
1.0 & 3 & 5.5104 & $3.4930 \pm 0.2802$ & 3.0616 \\
1.0 & 10 & 5.5104 & $4.2263 \pm 1.1971$ & 3.1754 \\
1.0 & 20 & 5.5104 & $3.7196 \pm 0.4081$ & 3.0461 \\
\hline
\end{tabular}


Table 8: Experiment results (test errors) on the Parkinson's - Motor dataset.

\begin{tabular}{rrrrr}
$\varphi$ & $n$ & NBR & CSO-NBR(mean) & CSO-NBR(best) \\
\hline 0.0 & 3 & 10.8869 & $7.8192 \pm 0.1168$ & 7.6027 \\
0.0 & 10 & 10.8869 & $8.2723 \pm 0.5394$ & 7.4407 \\
0.0 & 20 & 10.8869 & $\mathbf{7 . 6 2 9 8} \pm \mathbf{0 . 8 5 8 9}$ & $\mathbf{6 . 5 9 6 8}$ \\
0.1 & 3 & 10.8869 & $8.2869 \pm 0.3250$ & 7.8042 \\
0.1 & 10 & 10.8869 & $8.4412 \pm 0.3450$ & 8.0629 \\
0.1 & 20 & 10.8869 & $8.4356 \pm 0.4726$ & 7.9741 \\
0.5 & 3 & 10.8869 & $7.9885 \pm 0.2380$ & 7.5894 \\
0.5 & 10 & 10.8869 & $7.8277 \pm 0.2719$ & 7.2337 \\
0.5 & 20 & 10.8869 & $8.0523 \pm 0.4004$ & 7.5780 \\
1.0 & 3 & 10.8869 & $7.8931 \pm 0.3850$ & 7.3222 \\
1.0 & 10 & 10.8869 & $7.7304 \pm 0.3464$ & 7.2379 \\
1.0 & 20 & 10.8869 & $8.0553 \pm 0.2759$ & 7.6781 \\
\hline
\end{tabular}

Table 9: Experiment results (test errors) on the Parkinson's - Total dataset.

\begin{tabular}{lrrrr}
\hline$\varphi$ & $n$ & NBR & CSO-NBR(mean) & CSO-NBR(best) \\
\hline 0.0 & 3 & 18.9802 & $9.6309 \pm 0.1369$ & 9.4488 \\
0.0 & 10 & 18.9802 & $9.6215 \pm 0.2970$ & $\mathbf{9 . 1 1 0 4}$ \\
0.0 & 20 & 18.9802 & $9.6947 \pm 0.3364$ & 9.2157 \\
0.1 & 3 & 18.9802 & $\mathbf{9 . 5 5 3 7} \pm \mathbf{0 . 1 0 0 6}$ & 9.3631 \\
0.1 & 10 & 18.9802 & $9.9890 \pm 0.1606$ & 9.7112 \\
0.1 & 20 & 18.9802 & $9.9324 \pm 0.1307$ & 9.7260 \\
0.5 & 3 & 18.9802 & $9.5729 \pm 0.1130$ & 9.4543 \\
0.5 & 10 & 18.9802 & $9.7709 \pm 0.2050$ & 9.4648 \\
0.5 & 20 & 18.9802 & $9.8207 \pm 0.1653$ & 9.6467 \\
1.0 & 3 & 18.9802 & $9.5969 \pm 0.1199$ & 9.4083 \\
1.0 & 10 & 18.9802 & $9.6908 \pm 0.2172$ & 9.2791 \\
1.0 & 20 & 18.9802 & $9.8576 \pm 0.2160$ & 9.3910 \\
\hline
\end{tabular}

Table 10: Experiment results (test errors) on the Appliance Energy dataset.

\begin{tabular}{lrrrrr}
\hline$\varphi$ & $n$ & NBR & CSO-NBR(mean) & $p$ value & CSO-NBR(best) \\
\hline 0.0 & 3 & 96.1397 & $95.6540 \pm 5.1323$ & 0.3858 & 91.0486 \\
0.0 & 10 & 96.1397 & $98.6674 \pm 10.4532$ & 0.7680 & 90.2488 \\
0.0 & 20 & 96.1397 & $94.1240 \pm 2.0230$ & 0.0059 & 91.7284 \\
0.1 & 3 & 96.1397 & $92.5080 \pm 2.3186$ & 0.0004 & $\mathbf{8 8 . 9 9 6 4}$ \\
0.1 & 10 & 96.1397 & $110.8083 \pm 15.0077$ & 0.9935 & 91.6589 \\
0.1 & 20 & 96.1397 & $119.6089 \pm 26.7894$ & 0.9891 & 92.8323 \\
0.5 & 3 & 96.1397 & $91.2650 \pm 0.7496$ & 0.0000 & 89.9534 \\
0.5 & 10 & 96.1397 & $116.4989 \pm 20.3208$ & 0.9943 & 95.5893 \\
0.5 & 20 & 96.1397 & $118.0088 \pm 17.2667$ & 0.9985 & 93.1136 \\
1.0 & 3 & 96.1397 & $\mathbf{9 0 . 9 1 9 8} \pm \mathbf{1 . 1 3 6 8}$ & 0.0000 & 89.9421 \\
1.0 & 10 & 96.1397 & $118.5223 \pm 8.2297$ & 1.0000 & 102.9101 \\
1.0 & 20 & 96.1397 & $123.2761 \pm 11.2777$ & 1.0000 & 109.8786 \\
\hline
\end{tabular}


Table 11: Experiment results (test errors) on the Survival dataset.

\begin{tabular}{rrrrrr}
$\varphi$ & $n$ & NBR & CSO-NBR $($ mean $)$ & $p$ value & CSO-NBR(best) \\
\hline 0.0 & 3 & 3.2272 & $3.0148 \pm 0.2732$ & 0.0181 & 2.6715 \\
0.0 & 10 & 3.2272 & $2.9216 \pm 0.3084$ & 0.0060 & 2.2261 \\
0.0 & 20 & 3.2272 & $\mathbf{2 . 7 3 3 3} \pm \mathbf{0 . 2 1 1 0}$ & 0.0000 & 2.3555 \\
0.1 & 3 & 3.2272 & $4.0539 \pm 0.9391$ & 0.9894 & 3.5272 \\
0.1 & 10 & 3.2272 & $3.7990 \pm 0.4212$ & 0.9990 & 2.9585 \\
0.1 & 20 & 3.2272 & $3.5180 \pm 0.5446$ & 0.9372 & 2.8164 \\
0.5 & 3 & 3.2272 & $2.7568 \pm 0.4411$ & 0.0041 & $\mathbf{2 . 1 7 8 3}$ \\
0.5 & 10 & 3.2272 & $3.7682 \pm 0.7155$ & 0.9798 & 2.5391 \\
0.5 & 20 & 3.2272 & $3.1950 \pm 0.2570$ & 0.3505 & 2.8708 \\
1.0 & 3 & 3.2272 & $2.7909 \pm 0.3210$ & 0.0010 & 2.3641 \\
1.0 & 10 & 3.2272 & $3.6973 \pm 0.7256$ & 0.9646 & 2.7918 \\
1.0 & 20 & 3.2272 & $3.4808 \pm 0.4700$ & 0.9389 & 2.6984 \\
\hline
\end{tabular}

Tables 4 to 11 summarise the results. The column names have the same interpretation as previously, and for each table, the best achieved test set errors (both averaged over ten runs and for a single run) have been bolded.

Firstly we examined the results in respect of the parameter values we were varying. The results show that in most cases, a low value of $\varphi$ (either 0.0 or 0.1 ) achieves the lowest mean test error. The only exceptions to this are the Red Wine dataset and the Appliance Energy dataset for which a higher $\varphi$ of 1.0 leads to the best overall results. However, runs with lower $\varphi$ still significantly outperform the baseline on the Appliance Energy dataset.

The results are not as clear-cut with the dataset size parameter $n$ however. There is no consistent pattern of optimal artificial dataset size across the problems. For example, the Survival dataset appears to require a large artificial dataset with $n=20$. When $n$ is reduced, performance degrades significantly. Conversely, for the Energy Efficiency - Heating dataset, a very small number of artificial examples (i.e. $n=3$ ) is preferred and increasing this reduces accuracy.

The takeaway from the analysis is that while there is no clearly optimal artificial dataset size, a low value of $\varphi$ (either 0.0 or 0.1 ) appears to be a good default in most cases. Specific problems may benefit from gradually increasing $\varphi$ if performance is not ideal.

The next step in our examination was to perform statistical significance tests comparing NBR and CSONBR for a more rigorous comparison. Similar to the previous section, we performed single sample $t$-tests comparing the test set errors of CSO-NBR against the single result obtained by the deterministic NBR algorithm, for each set of ten runs. For the first six problems, the results of the tests unanimously showed CSO-NBR significantly outperforming NBR with exceptionally low $p$ values (i.e. $p \ll 0.0001$ ), but higher $p$ values were exhibited in the cases of the latter two (Appliance Energy and Survival) problems. We therefore include $p$ values in the tables of results only for the latter two regression problems, since these are the interesting results where the significance varies considerably.

Finally, we also ran standard with default parameters and parameter optimised GPR on the same datasets. The test set errors are shown in Table 12, and are mainly provided for context: the main focus of our comparison is between NBR and CSO-NBR in this section. To summarise, CSO-NBR significantly improves NBR's performance, to the point that it is at competitive with, and sometimes better than, LR, and occasionally better than state-of-the-art black box methods such as GPR. 
Table 12: Test error of linear regression (LR) and parameter-optimised Gaussian processes regression (GPR) on the datasets used in the experiment.

\begin{tabular}{lrr}
\hline Dataset & GPR & LR \\
\hline Wine Quality - Red & 0.66 & 0.68 \\
Wine Quality - White & 0.84 & 0.83 \\
Energy Efficiency - Heating & 2.08 & 3.78 \\
Energy Efficiency - Cooling & 2.44 & 3.54 \\
Parkinson's - Motor & 16.20 & 7.07 \\
Parkinson's - Total & 11.97 & 9.33 \\
Appliance Energy & 177.20 & 92.55 \\
Survival & 2.47 & 5.16 \\
\hline
\end{tabular}

\section{Analysis}

In this section, we explore the performance of CSO-NBR on a very simple two dimensional dataset with known properties, in an attempt to understand the interaction between the original NBR algorithm and our enhanced version.

The artificial dataset we employ in this section is generated using the "peak" algorithm in the R package MLBench (Leisch and Dimitriadou, 2010). Figure1 1 depicts the ground truth from which the data is generated. The data is drawn from a two dimensional normal distribution centred on the origin and scaled to a maximum height of 25. Points are sampled uniformly from the distribution within a circle of radius three about the origin. To generate a training dataset, we sampled 100 such points. A test set was then generated from a further 100 random samples. Each example in the dataset is a tuple of the form $(x, y, z)$ where $z$ is the target to predict.

We note the following points about this artificial regression problem:

1. The target variable $z$ is a non-linear function of the two predictors $x$ and $y$. This property of the data makes the problem difficult for simple linear model learners such as LR. This fact is demonstrated by the performance achieved using the LR algorithm to predict the $z$ values on both the training and test datasets after model learning using the training data: LR achieves a very poor RMSE score of 8.4 on the training data and 8.7 on the test data.

2. The predictive features $x$ and $y$ are not conditionally independent given the predictive target $z$, which violates naive Bayes' conditional independence assumption. Once $z$ is known, the potential values for $(x, y)$ are distributed around the perimeter of a circle centred on the origin. Thus, knowing the value of $x$ limits the locations for $y$ to two possible values. In contrast to LR, NBR achieves RMSE scores of 1.4 and 2.1 on the two data splits respectively, which is a major improvement but still not ideal.

To visualise the model that NBR learns from the training data, we constructed the contour plot depicted in Figure 2, Data from the plot was generated by dividing the $[-3 \ldots 3] \times[-3 \ldots 3]$ square centred on the origin into a $120 \times 120$ grid. At each grid point, we used the NBR model to make a prediction. Thus, $121 \times 121$ predictions were made in total, and the contour plot shows the predicted values. Figure 2 also depicts the 100 randomly sampled training data points used to construct the model as points on the contour plot.

Next, we ran the CSO-NBR algorithm ten times with the same settings as before $(s=100, \varphi=0.1$, $\left.t_{\max }=1000, n=10\right)$ and selected the resulting single best artificial training dataset from across the ten runs. The average model achieved an RMSE of $0.7 \pm 0.12$ on the test data. The best model, on the other 


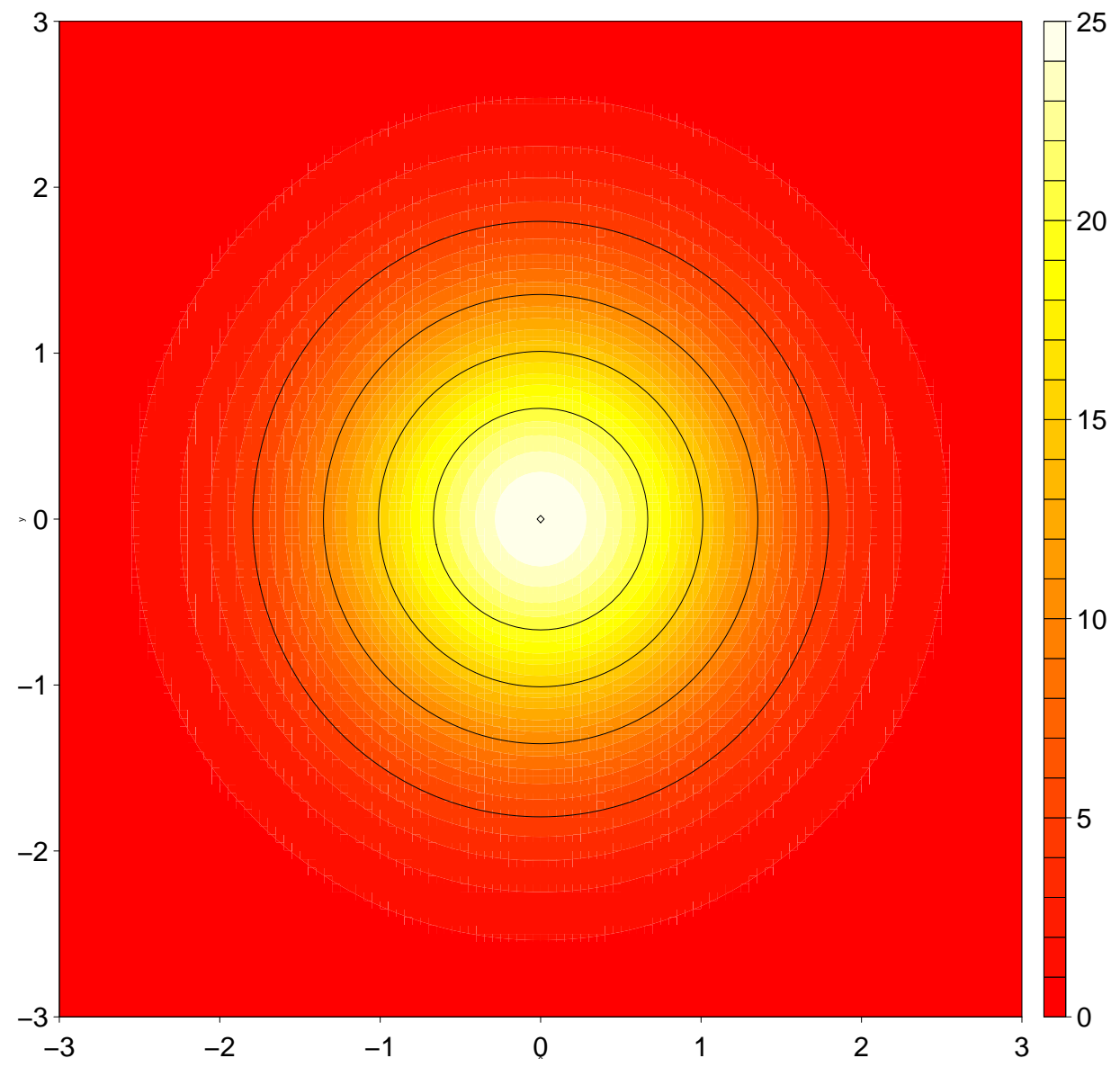

Figure 1: Contour plot showing a two-dimensional distribution from which artificial data points were sampled. The distribution is defined by the equation $z=25 e^{-\frac{1}{2} r^{2}}$ where $r$ is the distance of each sample point $(x, y)$ from the origin. 


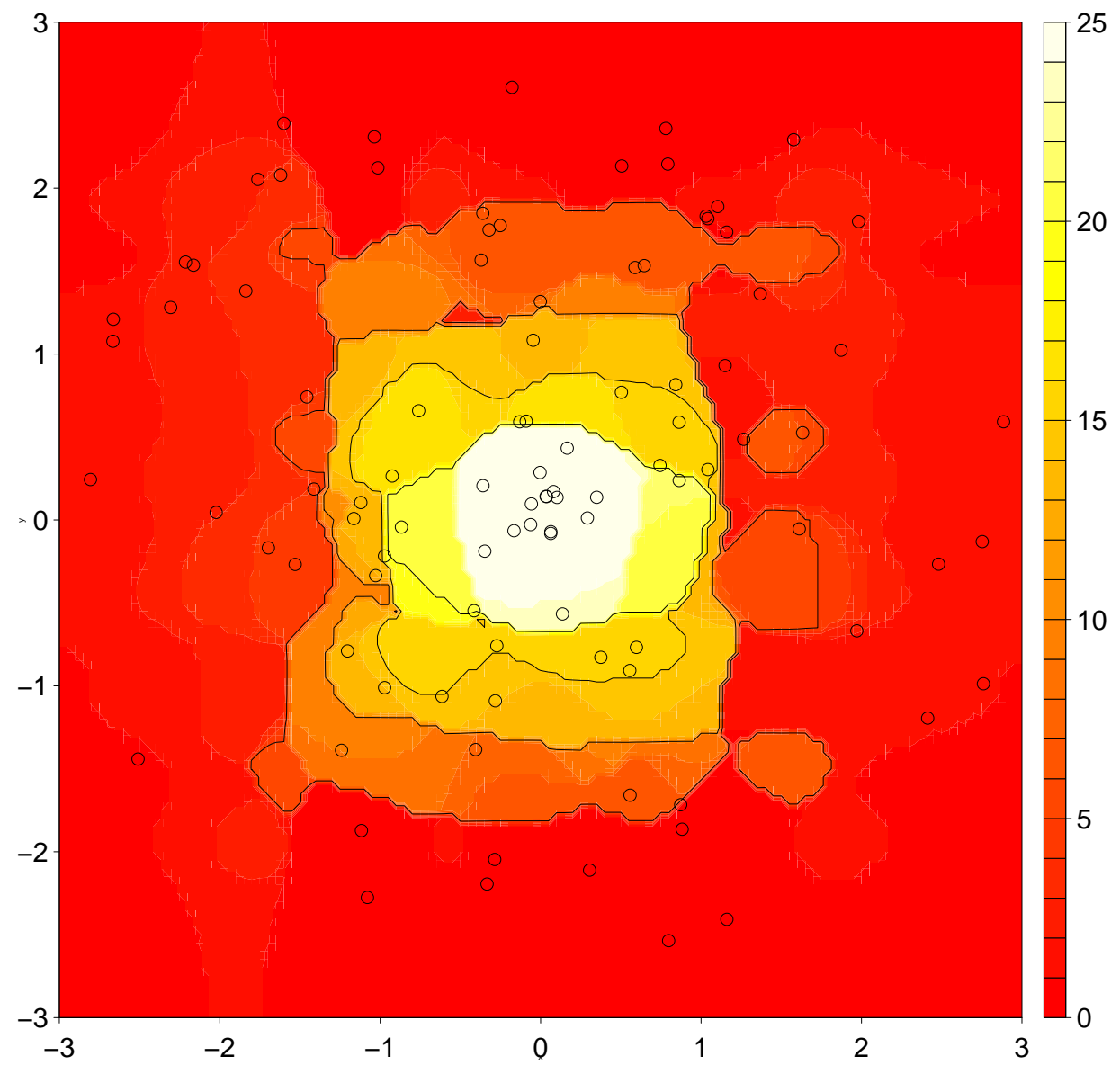

Figure 2: Predicted distribution using the NBR algorithm trained using the 100 training examples. The examples are depicted in the figure as points, and were generated using the R package MLBench (Leisch and Dimitriadou, 2010). 


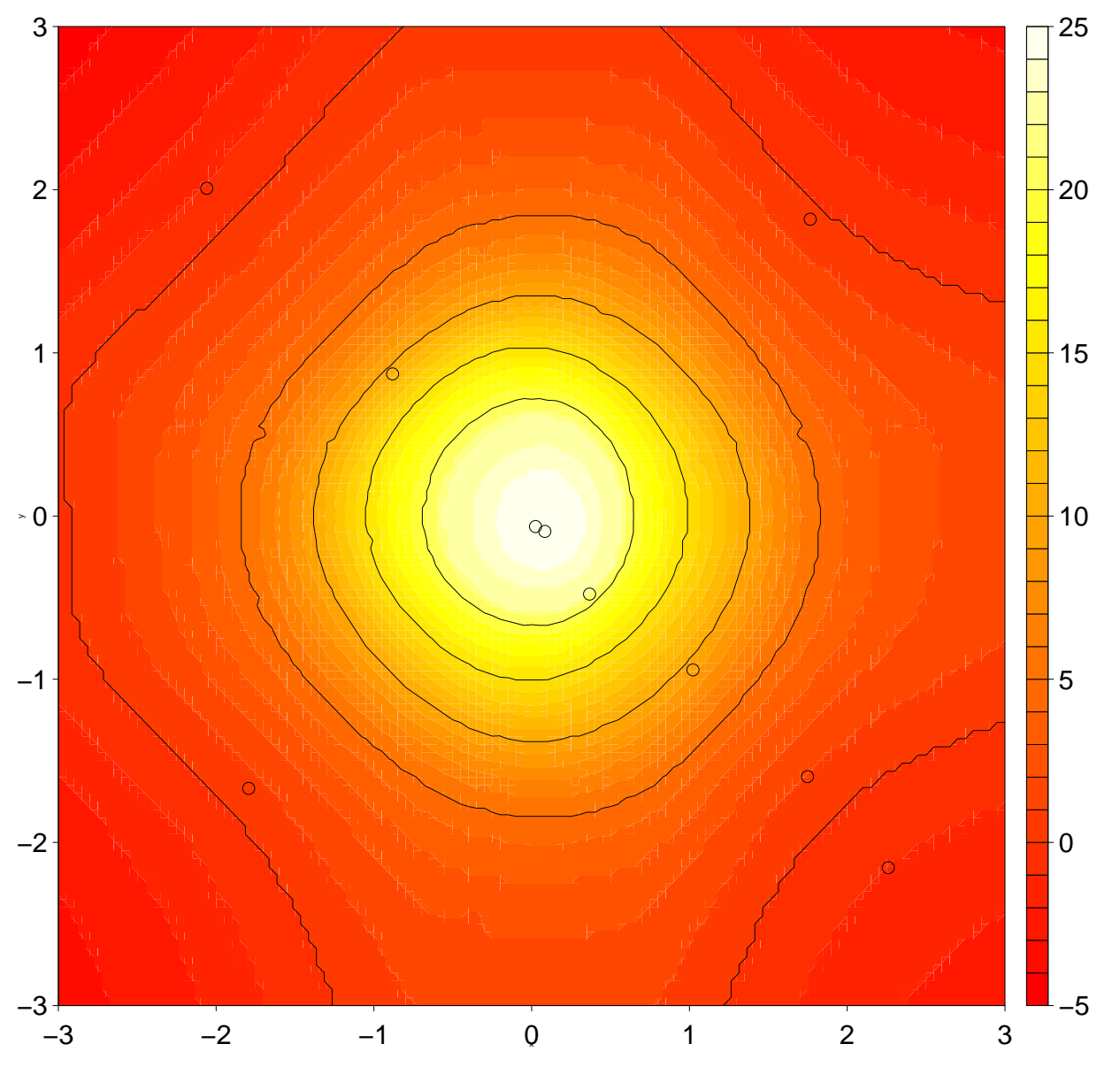

Figure 3: Predicted distribution using the CSO-NBR algorithm trained using ten optimised artificial training examples. The examples are depicted in the figure as points.

hand, achieved an RMSE score of 0.4 on the training data and 0.5 on the test data - both the average and best result are a substantial improvement on both LR and standard NBR.

Figure 3 depicts the predictions made by the CSO-NBR model along with the $(x, y)$ coordinates of the ten artificial data points in the best artificial datasets used to build the model. Table 13 further shows the actual artificial data points themselves. We can make several observations from the contour plots of the predictions and the table. Firstly, as Figure 2 clearly shows, the NBR algorithm by itself models the ground truth to a reasonable degree. The predicted $z$ values are higher the closer that $(x, y)$ is to the origin, which is what is expected. However, the circular symmetricity of the distribution in Figure 2 is not preserved well, as the contours have an obvious "squarish" appearance. There also appears to be a high degree of noise in the contours, suggesting that the model is not generalising well and instead overfitting the training data noise.

In contrast, the CSO-NBR predictions as depicted in Figure 3 produce a much cleaner, more symmetric, and less noisy predicted distribution. The prediction agrees well with the ground truth distribution in Figure 1. which in turn explains the low RMSE scores achieved in the experiments we performed. Of interest is the layout of the artificial data points across the distribution of predictions. Clearly, the positions of the artificial points have been highly optimised with the majority of the points lying approximately on the 
Table 13: Artificial data (rounded to 2 d.p.) used to train the model whose predictions are depicted in Figure 3

\begin{tabular}{rrr}
\hline$x$ & $y$ & $z$ \\
\hline 1.75 & -1.60 & 0.72 \\
0.08 & -0.09 & 30.89 \\
1.02 & -0.94 & 9.87 \\
0.02 & -0.06 & -2.81 \\
2.26 & -2.15 & -5.22 \\
-0.88 & 0.87 & 11.73 \\
0.37 & -0.48 & 17.51 \\
1.76 & 1.82 & 1.58 \\
-2.06 & 2.01 & -2.91 \\
\hline
\end{tabular}

negative diagonal $y=-x$ and the remaining two points lying on the opposite diagonal. This configuration of points, when used as training data for the NBR algorithm, produces superior predictions and indicates that only a small amount of training data is required to effectively model this problem.

We additionally performed some time experiments with NBR and CSO-NBR on the artificial data. Over ten runs on an Intel Core i7-6700K CPU running at 4.00GHz with $64 \mathrm{~GB}$ of RAM and using the same settings as above, the mean training time for NBR was 0.017 seconds, compared to 98.97 seconds for CSO-NBR. CSO-NBR made use of six cores while our NBR implementation is single-threaded. When we increased the dimensionality of problem from two to ten dimensions (by creating a new artificial dataset with the same number of examples), the mean runtimes of both algorithms was 0.097 seconds vs. 428.23 seconds. For the 30-dimensional peak problem, the runtimes were 0.28 seconds and 1241.99 seconds.

Clearly, the training time for CSO-NBR is much higher than NBR (which is to be expected, since each run of CSO-NBR builds approximately 500,000 NBR models), and moreover, the training time increases at a rate that is worse than linear with the dimensionality of the regression problem. We expect that the training for CSO-NBR, in practice, would be even worse, since in these experiments we have held the number of iterations constant. With an increase in dimensionality, however, comes a corresponding increase in the size of the search space for CSO, and therefore to properly solve the problem CSO will need significantly more iterations. Mitigating this, however, is the fact that CSO is an "embarrassingly parallel" algorithm: i.e. doubling the number of cores available should effectively halve the runtime.

\section{Conclusion}

In summary, we have described a method for enhancing the predictive accuracy of naive Bayes for regression. The approach employs "real" training data only indirectly in the machine learning pipeline, as part of a fitness function that in turn is used to optimise a small artificial surrogate training dataset. The naive Bayes model is constructed using the artificial data instead of the real training data. Our evaluation has demonstrated that the method often produces superior regression models in terms of generalisation error.

The primary advantages of this idea are twofold. In its original formulation, naive Bayes typically underperforms other regression models. However, the algorithm does have certain specific advantages such as being simple and interpretable, and interpretable models are important in many areas (e.g. medicine) where understanding the rules used for prediction is important. Finding new methods to enhance the accuracy of naive Bayesian models is therefore important, and we have described one means of doing so.

Several interesting questions are raised by this research, which may form a basis for future work. For 
example, how hard is this problem when framed as an optimisation problem, from a fitness landscape perspective? "Easy" problems in evolutionary computation are usually those with a high correlation between the fitness of solutions and their distance to the global optima. "Hard" problems, on the other hand, have a much lower or even negative (in the case of deception) correlation.

Another question concerns the behaviour of our proposed algorithm when a base algorithm quite different to NBR is employed. How exactly does CSO's behaviour change with the base model? Can we replace NBR with a full Bayesian network or a decision tree ensemble and still get generalisation improvements? Do we need to use larger or smaller artificial datasets to make our approach work with other base algorithms?

There is also the issue of how to select the optimal artificial dataset size for the problem at hand. Our results indicate that there is no single value of $n$ that is a good default in most cases. Some problems require a larger $n$, and others a smaller $n$. How, then, can the correct $n$ be chosen? A potential but expensive answer to this question is to run the algorithm multiple times with different artificial dataset sizes and attempt to hone in on the best one. However, other more intelligent approaches (for example, greedily adding one optimised example to the artificial dataset at a time until no more improvements are possible) are certainly possible and may work.

Understanding how and why the algorithm works theoretically is a vital next step. Recently, efficient algorithms for computing influence functions have been proposed in the literature (Koh and Liang, 2017).

Influence functions relate the change in weight of a training example to training loss, and are therefore a means of assessing the impact of each individual example on predictive performance. Are these ideas applicable here? If so, we could use them to understand the relationship betweens real and fake training data, and model predictions.

Research involving surrogate models for evolutionary algorithms may also yield improvements. If the fitness of a model trained from a dataset can be estimated approximately using a different model, then the optimisation speed could potentially speed up significantly. Again, influence functions may be useful other; other surrogate model approaches could also be explored.

Finally, is it possible that useful information might be discovered in these small highly-optimised artificial datasets, in the same way that rules and other knowledge can be typically discovered by inspecting normal machine learning models? Figure 3 shows that the optimised artificial data points are uniformly distributed across the space of examples. Would it be possible to take an artificial dataset like this and use a different type of analysis to extract useful knowledge about the problem domain?

\section{References}

Agrawal R, Imielinski T, Swami A (1993) Database mining: A performance perspective. IEEE Transactions on Knowledge and Data Engineering 5(6):914-925

Bonyadi M, Michalewicz Z (2017) Particle swarm optimization for single objective continuous space problems: A review. Evolutionary Computation 25(1):1-54

Brameier M, Banzhaf W (2007) Linear Genetic Programming. Springer

Breiman L (1996) Bagging predictors. Machine Learning 24(2):123-140

Breiman L (2001) Random forests. Machine Learning 45(1):5-32 
Candanedo L, Feldheim V, Deramix D (2017) Data driven prediction models of energy use of applicances in a low energy house. Energy and Buildings 140:81-97

Chawla N, Bowyer K, Hall L, Kegelmeyer W (2002) Synthetic minority over-sampling technique. Journal of Artificial Intelligence Research 16:321-357

Cheng R, Jin Y (2015) A competitive swarm optimizer for large scale optimization. IEEE Transactions on Cybernetics 45(2):191-204

Cortez P, Cerdeira A, Almeida F, Matos T, Reis J (2009) Modeling wine preferences by data mining from physicochemical properties. Decision Support Systems 47(4):547-553

Escalante H, Mendoza K, Graff M, Morales-Reyes A (2013) Genetic Programming of Prototypes for Pattern Classification, Springer Berlin Heidelberg, Berlin, Heidelberg, pp 100-107

Frank E, Trigg L, Holmes G, Witten I (2000) Naive Bayes for regression. Machine Learning 41:5-25

Frank E, Hall M, Witten I (2016) The WEKA Workbench. Online Appendix for "Data Mining: Practical Machine Learning Tools and Techniques", 4th edn, Morgan Kaufmann

Goodfellow I, Pouget-Abadie J, Mirza M, Xu B, Warde-Farley D, Ozair S, Courville A, Bengio Y (2014) Generative adversarial nets. arXiv:14062661

Greene C, Hill D, Moore J (2009) Environmental noise improves epistasis models of genetic data discovered using a computational evolution system. In: Proc. GECCO’09, pp 1785-1786

Guozhong A (1996) The effects of adding noise during backpropagation training on generalisation performance. Neural Computation 8(3):643-674

Impedovo S, Mangini F, Barbuzzi D (2014) A novel prototype generation technique for handwriting digit recognition. Pattern Recognition 47(3):1002 - 1010

Kapur A, Marwah K, Alterovitz G (2016) Gene expression prediction using low-rank matrix completion. BMC Bioinformatics 17:243

Kennedy J, Eberhart R, Shi Y (2001) Swarm Intelligence. The Morgan Kaufmann Series in Artificial Intelligence, Morgan Kauffman

Koh PW, Liang P (2017) Understanding black-box predictions via influence functions. In: Precup D, Teh YW (eds) Proceedings of the 34th International Conference on Machine Learning, PMLR, International Convention Centre, Sydney, Australia, Proceedings of Machine Learning Research, vol 70, pp 1885-1894, URL http://proceedings.mlr.press/v70/koh17a.html

Leisch F, Dimitriadou E (2010) MLbench: Machine Learning Benchmark Problems. v. 2.1-1 edn

Lenz G, Wright G, Dave S, Xiao W, Powell J, Zhao H, Xu W, Tan B, Goldschmidt N, Iqbal J, Vose J, Bast M, Fu K, Weisenburger D, Greiner T, Armitage J, Kyle A, May L, Gascoyne R, Connors J, Troen G, Holte H, Kvaloy S, Dierickx D, Verhoef G, Delabie J, Smeland E, Jares P, Martinez A, Lopez-Guillermo A, Montserrat E, Campo E, Braziel R, Miller T, Rimsza L, Cook J, Pohlman B, Sweetenham J, Tubbs R, Fisher R, Hartmann E, Rosenwald A, Ott G, Muller-Hermelink HK, Wrench D, Lister T, Jaffe E, Wilson W, Chan W, Staudt L (2008) Stromal gene signatures in large-b-cell lymphomas. New England 
Journal of Medicine 359(22):2313-2323, DOI 10.1056/NEJMoa0802885, URL http://dx.doi.org/10. 1056/NEJMoa0802885, pMID: 19038878, http://dx.doi.org/10.1056/NEJMoa0802885

Lòpez V, Triguero I, Carmona C, Garcia S, Herrara F (2014) Addressing imbalanced classification with instance generation techniques: IPADE-ID. Neurocomputing 126(15-28)

Mayo M, Sun Q (2014) Evolving artificial datasets to improve interpretable classifiers. In: 2014 IEEE Congress on Evolutionary Computation (CEC), pp 2367-2374

Triguero I, Derrac J, Garcia S, Herrera F (2011) A taxonomy and experimental study on prototype generation for nearest neighbor classification. IEEE Trans on Systems, Man and Cybernetics - Part C: Applications and Reviews 42(1):86-100

Tsanas A, Xifara A (2012) Accurate quantitative estimation of energy performance of residential buildings using statistical machine learning tools. Energy and Buildings 49:560-567

Tsanas A, Little M, McSharry P, Ramig L (2010) Accurate telemonitoring of Parkinson's disease progression by non-invasive speech tests. IEEE Transactions on Biomedical Engineering 57:884-893

Urbanowicz R, Sinnot-Armstrong N, Moore J (2011) Random artificial incorporation of noise in a learning classifier system environment. In: Proc. GECCO'11, pp 369-374

Vincent P, Larochelle H, Bengio Y, Manzagol P (2008) Extracting and composing robust features with denoising autoencoders. Proc 25th International Conference on Machine Learning (ICML'08)

Wang Y, Witten I (1997) Induction of model trees for predicting continuous classes. In: Poster papers of the 9th European Conference on Machine Learning, Springer

Wang Z (2015) bujar: Buckly-James regression for survival data with high dimensional covariates. Tech. rep.

Zhong K, Guo R, Kumar S, Yan B, S D, Dhillon I (2017) Fast classification with binary prototypes. In: Proceedings of the 20th International Conference on Artificial Intelligence and Statistics, PMLR, vol 54, pp 1255-1263

\section{Appendix}


Table 14: Results of running SPSO and SPSO-NBR on the datasets used in the first experiment with $s=50$ and $t_{\max }=1000$. Shown are the test errors. Bolded figures in the SPSO-NBR(mean) column indicate a better result than the corresponding CSO-NBR(mean) result.

\begin{tabular}{lrrr}
\hline Dataset & NBR & SPSO-NBR(mean) & SPSO-NBR(best) \\
\hline auto93 & 5.41 & $7.28 \pm 2.53$ & 4.46 \\
autoHorse & 12.92 & $19.53 \pm 2.84$ & 16.56 \\
\hline autoMpg & 3.43 & $3.56 \pm 0.28$ & 3.04 \\
autoPrice & 2375.45 & $2418.18 \pm 217.03$ & 2171.16 \\
\hline baskball & 0.11 & $0.10 \pm 0.01$ & 0.08 \\
bodyfat & 1.68 & $1.33 \pm 1.24$ & 0.70 \\
\hline bolts & 7.92 & $\mathbf{9 . 4 8} \pm \mathbf{2 . 2 2}$ & 5.82 \\
cholesterol & 61.76 & $\mathbf{5 2 . 8 0} \pm \mathbf{2 . 0 4}$ & 49.40 \\
\hline cleveland & 1.05 & $0.86 \pm 0.07$ & 0.79 \\
\hline cloud & 0.43 & $0.55 \pm 0.20$ & 0.37 \\
\hline cpu & 114.65 & $56.95 \pm 32.54$ & 19.48 \\
\hline detroit & 89.18 & $63.51 \pm 21.85$ & 38.91 \\
\hline echoMonths & 12.67 & $\mathbf{1 2 . 2 0} \pm \mathbf{1 . 1 4}$ & 10.74 \\
elusage & 17.73 & $17.84 \pm 2.32$ & 14.93 \\
fishcatch & 209.45 & $80.08 \pm 16.97$ & 53.79 \\
fruitfly & 19.80 & $\mathbf{1 6 . 8 9} \pm \mathbf{0 . 9 3}$ & 15.40 \\
\hline gascons & 17.63 & $5.54 \pm 2.63$ & 3.04 \\
housing & 6.32 & $4.53 \pm 0.87$ & 3.95 \\
\hline hungarian & 0.36 & $0.34 \pm 0.02$ & 0.32 \\
longley & 552.15 & $910.21 \pm 504.25$ & 408.73 \\
\hline lowbwt & 559.99 & $479.54 \pm 30.36$ & 455.66 \\
mbagrade & 0.30 & $0.33 \pm 0.02$ & 0.31 \\
\hline meta & 378.52 & $511.75 \pm 243.39$ & 184.12 \\
pbc & 1049.74 & $1026.42 \pm 46.36$ & 964.19 \\
\hline pharynx & 354.80 & $330.98 \pm 31.81$ & 303.07 \\
pollution & 62.31 & $66.89 \pm 26.40$ & 43.90 \\
\hline pwLinear & 2.49 & $2.24 \pm 0.17$ & 2.04 \\
quake & 0.27 & $0.20 \pm 0.00$ & 0.19 \\
\hline schlvote & 1956688.47 & $0.82 \pm 0.02$ & 0.78 \\
\hline sensory & 0.94 & $0.88 \pm 0.18$ & 0.67 \\
servo & 1.04 & $\mathbf{1 1 . 4 3} \pm 20.98$ & 2.43 \\
\hline sleep & 4.09 & $\mathbf{2 . 3 5} \pm \mathbf{0 . 2 5}$ & 656.73 \\
\hline strike & & 145.96 \\
\hline veteran & 164.17 & $\mathbf{1 0 . 2 1}$ \\
\hline vineyard & 1.33 & & \\
\hline
\end{tabular}


Table 15: Results of running SPSO and SPSO-NBR on the datasets used in the first experiment with $s=100$ and $t_{\max }=500$. Shown are the test errors. Bolded figures in the SPSO-NBR(mean) column indicate a better result than the corresponding CSO-NBR(mean) result.

\begin{tabular}{lrrr}
\hline Dataset & NBR & SPSO-NBR(mean) & SPSO-NBR(best) \\
\hline auto93 & 5.41 & $6.58 \pm 1.23$ & 5.07 \\
autoHorse & 12.92 & $17.08 \pm 2.74$ & 12.61 \\
\hline autoMpg & 3.43 & $3.55 \pm 0.22$ & 3.19 \\
autoPrice & 2375.45 & $2524.65 \pm 536.17$ & 2217.93 \\
\hline baskball & 0.11 & $0.10 \pm 0.04$ & 0.08 \\
\hline bodyfat & 1.68 & $0.70 \pm 0.10$ & 0.53 \\
\hline bolts & 7.92 & $\mathbf{9 . 1 1} \pm \mathbf{3 . 4 1}$ & 5.12 \\
\hline cholesterol & 61.76 & $\mathbf{5 4 . 3 7} \pm \mathbf{2 . 4 7}$ & 50.80 \\
\hline cleveland & 1.05 & $0.84 \pm 0.08$ & 0.75 \\
\hline cloud & 0.43 & $0.55 \pm 0.16$ & 0.34 \\
\hline cpu & 114.65 & $77.61 \pm 36.68$ & 25.26 \\
\hline detroit & 89.18 & $\mathbf{4 5 . 2 7} \pm \mathbf{1 1 . 8 9}$ & 28.58 \\
\hline echoMonths & 12.67 & $\mathbf{1 1 . 8 9} \pm \mathbf{0 . 7 5}$ & 11.25 \\
elusage & 17.73 & $\mathbf{1 7 . 3 5} \pm \mathbf{2 . 3 0}$ & 13.38 \\
\hline fishcatch & 209.45 & $92.72 \pm 66.22$ & 55.98 \\
fruitfly & 19.80 & $18.02 \pm 1.25$ & 16.48 \\
\hline gascons & 17.63 & $5.93 \pm 2.48$ & 2.39 \\
\hline housing & 6.32 & $4.10 \pm 0.13$ & 3.92 \\
\hline hungarian & 0.36 & $0.34 \pm 0.02$ & 0.31 \\
\hline longley & 552.15 & $1061.36 \pm 1367.85$ & 372.86 \\
\hline lowbwt & 559.99 & $\mathbf{5 2 2 . 2 8} \pm \mathbf{5 9 . 4 5}$ & 466.73 \\
\hline mbagrade & 0.30 & $0.34 \pm 0.03$ & 0.32 \\
\hline meta & 378.52 & $566.25 \pm 316.02$ & 97.00 \\
pbc & 1049.74 & $\mathbf{1 0 0 9 . 4 7} \pm \mathbf{5 7 . 8 5}$ & 919.29 \\
\hline pharynx & 354.80 & $\mathbf{3 2 5 . 7 0} \pm \mathbf{4 4 . 7 4}$ & 272.44 \\
pollution & 62.31 & $57.13 \pm 15.40$ & 44.13 \\
\hline pwLinear & 2.49 & $2.26 \pm 0.27$ & 2.01 \\
\hline quake & 0.27 & $0.20 \pm 0.00$ & 0.19 \\
\hline schlvote & 1956688.47 & $0.83 \pm 0.02$ & 0.80 \\
\hline sensory & 0.94 & $\mathbf{0 . 7 6} \pm \mathbf{0 . 1 2}$ & 0.60 \\
\hline servo & 1.04 & $4.79 \pm 3.24$ & 2.55 \\
\hline sleep & 4.09 & $\mathbf{6 6 8 . 9 2} \pm \mathbf{1 3 . 4 4}$ & 655.56 \\
\hline strike & 696.60 & $\mathbf{1 7 3 . 8 3} \pm \mathbf{3 0 . 1 5}$ & 140.91 \\
\hline veteran & 164.17 & $\mathbf{2 . 6 1 2}$ & 2.11 \\
\hline vineyard & 1.33 & & \\
\hline
\end{tabular}

